\title{
Fabrication and Composition Control of NiTi Shape Memory Thin Films for Microactuators
}

David J. Getchel and Richard N. Savage

Materials Engineering Department, California Polytechnic State University, San Luis Obispo, CA 93407, U.S.A.

\begin{abstract}
Microactuators fabricated with NiTi thin films take advantage of the shape memory effect's large energy density $\left(\sim 5-10\right.$ joules $\left./ \mathrm{cm}^{3}\right)$ and high strain recovery $(\sim 8 \%)$. Microelectromechanical Systems (MEMS) designed with these actuators can serve as biosensors, micro-fluidic pumps or optical switches. However, the fundamental mechanical properties of these shape memory NiTi films have not been fully characterized with micro-scale test structures. Equiatomic NiTi thin films were deposited by co-sputtering NiTi and Ti targets with the intension of fabricating such test structures. Dual cathodes allowed direct control of the film composition by adjusting the Ti cathode power. Energy Dispersive Spectroscopy (EDS) quantified the film composition relative to pure standards. A thin $(\sim 50 \mathrm{~nm})$ chromium film on a pure silicon substrate created excellent film adhesion. Oxidized Si wafers did not bond with the $\mathrm{Cr}$ and NiTi films. This deposition method enabled control of film composition and the necessary adhesion.
\end{abstract}

\section{INTRODUCTION}

The shape memory effect (SME) of NiTi outperforms traditional electrostatic and thermal MEMS actuators when large range of motion and high actuation forces are desired [1]. NiTi is a challenging actuator material to use because the shape memory behavior is sensitive to deposition parameters and post deposition annealing [2]. The realization of NiTi MEMS actuators requires performance data from test structures similar in size to current MEMS devices. Previous tensile and diaphragm NiTi testing provide valuable film properties, but not on the micro-scale [3, 4]. Smaller test structures may expose how factors such as surface oxides or compositional variation in thickness [5] affect the mechanical and shape memory properties. Fabricating and testing a series of micro-scale NiTi test structures to examine how deposition parameters affect actuator performance motivated this study.

An ideal MEMS test structure would use scanning probe microscopy (SPM) instruments to deflect miniature thin film cantilevers. Nanoindenters are the ideal instruments for measuring force vs. displacement data from cantilevers because they are capable of measuring both tip displacement and tip force continuously during measurements [6]. Triangular cantilevers with optimized strain profiles [7] and pure tensile fixed beams [8] have also been fabricated for nanoindenter testing. A profilometer is a less expensive alternative with comparable displacement measurement precision, and is capable of measuring stylus displacement at fixed tip forces. Force displacement measurements of Ni cantilevers over silicon trenches have already been taken with a profilometer [9]. NiTi cantilevers have been made and tested with tiny weights [10], but they were made with very thick rolled sheet (50-95 microns). Bending micro-scale NiTi beams with a profilometer will confirm SMA actuator abilities for MEMS. 


\section{EXPERIMENTAL DETAILS}

NiTi films were deposited with DC magnetron co-sputtering onto un-heated $100 \mathrm{~mm}<100>$ silicon wafers. Independently controlled 2" diameter cathodes of equiatomic NiTi and Ti were chosen to create equiatomic NiTi thin films. Pure Argon gas (99.999\%) at 3.5 mTorr supplied the ions for sputtering. The pure $\mathrm{Ti}$ cathode compensated for the imbalanced sputtering rate of $\mathrm{Ni}$ and Ti. Substrate rotation during the deposition increased compositional uniformity. Piranha etchant $\left(\mathrm{H}_{2} \mathrm{SO}_{4}(96 \%): \mathrm{H}_{2} \mathrm{O}_{2}(30 \%), 9: 1\right.$ mixture by volume, at $70^{\circ} \mathrm{C}$ for 10 minutes) removed organic material from the wafers, and a buffered oxide etchant $\left(1\right.$ minute at $\left.25^{\circ} \mathrm{C}\right)$ stripped the native oxide prior to sputtering. Pre-sputtering both cathodes at $100 \mathrm{~W}$ for 30 minutes with a shutter covering the substrate removed target surface oxidation. Pre-sputtering also replicated a sputter-ion pump, and reduced the system base pressure below $2 \times 10^{-7}$ Torr. A $100 \mathrm{~nm}$ chromium film on top of the clean silicon served as an adhesion promoter, and will later serve as a mask layer for etching the silicon substrate. Varying the Ti cathode power through a series of NiTi film depositions changed the resulting film composition. Energy dispersive x-ray spectroscopy (EDS) analysis with pure metal calibration standards determined the film composition. Profilometer measurements of film thickness across the entire wafer determined the deposition rate to be $12 \AA$ per second.

\section{DISCUSSION}

The deposition process is capable of producing equiatomic NiTi thin film at a reasonable deposition rate. Table I. lists the values for Ti-rich and Ni-rich thin films and the power settings. Single watt adjustability of the Ti cathode power allows for precise composition control since a 2 at. \% shift in titanium content occurs over a $25 \mathrm{~W}$ range. Similar deposition parameters of $250 \mathrm{~W}$ to a NiTi target and $20 \mathrm{~W}$ to a Ti target were used by Shih [11] to obtain equiatomic films. The use of 3" targets by Shih, instead of 2" targets, most likely reduced Ti depletion and the need for high Ti cathode power. The NiTi film adhesion to silicon with a $\mathrm{Cr}$ adhesion layer is excellent. Figure 1(a), shows the torn silicon surface after removing the NiTi/Cr film. The adhesion between the metal layers and the pure silicon was stronger than the internal silicon bonds. Figure 1(b) shows how the poor adhesion between $\mathrm{NiTi} / \mathrm{Cr}$ and $\mathrm{SiO}_{2}$ allowed easy removal of the film without altering the substrate. Future processing of test structures should be robust because of the good composition control and the excellent adhesion.

Table I. NiTi thin film composition measurements at different Ti target power settings

\begin{tabular}{|c|c|c|c|}
\hline NiTi Power $(\mathrm{W})$ & Ti Power $(\mathrm{W})$ & At. \% Ti & At. \% Ni \\
\hline 250 & 50 & 48 & 52 \\
\hline 250 & 75 & 50 & 50 \\
\hline 250 & 100 & 52 & 48 \\
\hline
\end{tabular}




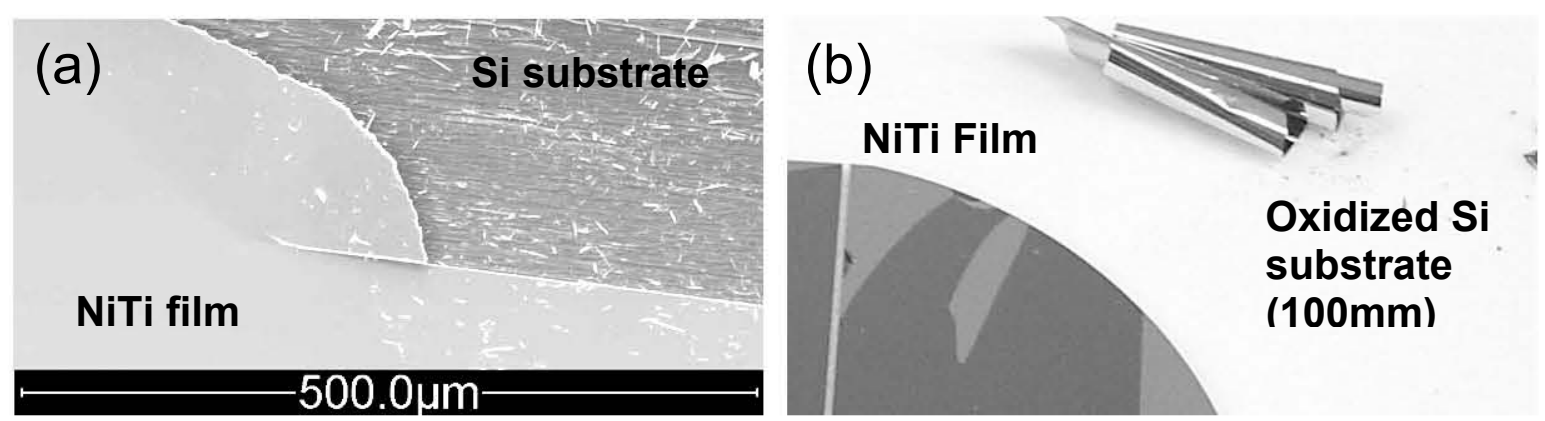

Figure 1. (a) Rough Si substrate created by removing NiTi film during wafer cleaving, and (b) easily removed NiTi film from oxidized Si wafer.

\section{CONCLUSIONS}

Co-sputtering of NiTi thin films with excellent deposition rate is possible with the described system and sputtering procedure. Pre-sputtering with a substrate shield is essential to eliminate oxygen content from the cathode surface and the vacuum chamber before deposition. The native oxide on silicon substrate must be removed to ensure good adhesion.

Future experimental work will include post deposition annealing and shape memory evaluation of the thin films. Differential Scanning Calorimetry (DSC) analysis will determine the start and finish transition temperatures for the low and high temperature crystalline phases. Grazing incidence XRD in conjunction with a temperature stage will verify the presence of each phase. Finally, measuring the displacement of micro-scale NiTi cantilevers while applying known loads at various temperatures will yield the power capabilities and fundamental mechanical properties. Evaluating the shape memory and mechanical behavior, as functions of temperature, should yield the efficiency of micro-scale NiTi actuators, and more importantly optimize film processing.

\section{REFERENCES}

1. P. Krulevitch, A. Lee, P. Ramsey, J. Trevino, J. Hamilton, M. Northrup, J. MEMS. Vol. 5 No. 4, 274 (1996)

2. Y. Fu, H. Du, S. Zhang, Surface and Coating Technology 167, 120 (2003)

3. A. Ishida, A. Takei, M. Sato, S. Miyazaki, Thin Solid Films 281-282, 337 (1996)

4. Y. Fu, H. Du, W. Huang, S. Zhang, M. Hu, Sen. \& Act. A 112, 397 (2004)

5. A. Ishida, M. Sato, Acta Materialia 51, 5578 (2003)

6. D. Son, J. Jeong, D. Kwon, Thin Solid Films 437, 183 (2003)

7. J. Florando, W. Nix, J. Mech. and Phys. of Solids 53, 621 (2005)

8. H. Espinosa, B. Prorok, M. Fischer, J. Mech. and Phys. of Solids 51, 49 (2003)

9. J. Luo, A. Flewitta, S. Spearingb, N. Flecka, W. Milnea, Mate. Lett. 58, 2307 (2004)

10. M. Kohl, D. Allen, T. Chen, S. Miyazaki, M. Schwörer, Mat. Sci. \& Eng. A 270, 146 (1999)

11. C. Shih, B. Lai, H. Kahn, S. Philips, A. Heuer, J. MEMS VOL. 10 NO. 1, 73 (2001) 